\title{
Quasar birthpangs observed?
}

\section{from C. Martin Gaskell}

BETWEen 5-10 per cent of high-redshift quasars show broad absorption lines arising from gas being expelled at speeds of up to 10 per cent of the speed of light. A recent study published in Astrophysical Journal 282, 33; 1984 by Cyril Hazard and collaborators leads to the suggestion that these so-called broad absorption line (BAL) quasars are actually quasars turning on for the first time.

Early studies showed that quasars are more common the further back in time one looks (that is, at higher redshifts). This produced confident predictions that surveys down to faint optical magnitudes and low radio-flux levels would turn up many highredshift quasars. However, despite the big advances over the past decade in radio and optical telescopes, and in particular in lowlight level detectors, the quasar redshift record has only crept from 3.53 in 1974 to 3.78 in 1982 . This lack of progress should be contrasted with the situation for more normal (non-quasar) galaxies over the same period, where the same advances in instrumentation have pushed the redshift record from 0.46 to 1.82 .

Where are the quasars with a redshift $(z)$ of greater than 3.8? As search techniques have been refined in recent years, it has become increasingly apparent that there is a real deficit of quasars with $z$ of greater than 3.2 , and this has led many people to suggest that by $z$ of say 3.5 we have already reached the epoch of quasar formation (and perhaps the epoch of galaxy formation as well). If this is true, then we might well expect high-redshift quasars to show spectroscopic signs of their youth.

One of the techniques being developed to search the sky for quasars (and other interesting objects) is objective-prism spectroscopy using the UK Schmidt telescope in Australia. The prism in front of the 48-inch Schmidt telescope produces millions of tiny spectra of extremely faint objects over a $6^{\circ} \times 6^{\circ}$ field of view. Hazard, one of the pioneers of this technique, and his collaborators report the discovery of nine new broad absorptionline quasars on objective-prism plates, expressing particular interest in their redshift distribution. Much of their paper is spent discussing the difficulties of recognizing BAL quasars on the UK Schmidt objective-prism plates. Normal quasars can be recognized relatively easily by their strong emission lines but the identification of quasars with broad absorption lines is much harder and in some cases almost impossible without follow-up spectroscopy on a large telescope. Their detectability is a strong function of their distance, because their redshifts govern what features fall within the observed wavelength region.
Hazard et al. expect to be able to detect BAL quasars in the redshift range of about $1.4-3$, with the range of about 2.1-2.3 being particularly favoured. Four of their nine BAL quasars fall in the favoured redshift range, but the five that lie outside this range all have higher redshifts. This is surprising, since one finds more non-BAL quasars in the redshift range of 1.4 - 2 than 2.4 - 3. It seems, therefore, that the fraction of quasars with broad absorption lines rises steeply at high redshifts.

This discovery is important. It leads Hazard et al. to suggest that BAL quasars are young quasars and that the broad absorption troughs are the result of some violent ejection during the first onset of quasar activity. In the course of time the BAL quasar will evolve into a more normal quasar. This contrasts with earlier suggestions that BAL quasars are either a special kind of quasar or, perhaps, are normal quasars viewed from a special angle. Whatever the cause of BAL quasars, the rise in the percentage of quasars showing broad absorption lines as redshift 3.5 is approached is strong support for 'something' having happened about that time.

Hazard et al. also point out that if many quasars pass through the broad absorption line phase, with heavy elements being rejected at high velocities, there could be a considerable influence on the pollution and dynamics of the intergalactic medium and the formation of galaxies. Full confirmation of these ideas will require more extensive studies of the frequency of BAL quasars among the quasar population as a whole and more follow-up spectroscopy of quasars found to have peculiar spectra on the UK Schmidt prism plates.

C. Martin Gaskell is in the Astronomy Department, University of Texas, Austin, Texas 78712, USA.

\section{Geochemistry}

\section{Multistage accretion and core formation of the Earth}

\section{from Shen-su Sun}

THERE are several competing hypotheses to explain the Earth's accretion from the solar nebula and the subsequent formation of its metal core within a period of 100 million years, about 4.6 thousand million years ago. Arguments are based on models of the development of the Solar System, studies of meteorites, experiments carried out under high temperature and pressure, and our knowledge of elemental abundances in the Earth's mantle. Specific processes of accretion and core formation have left their finger prints on the abundance patterns of elements with different affinities for silicates, metallic iron and sulphides. The strengths or weaknesses of various hypotheses can therefore often be exposed when accurate abundance data for certain critical elements become available. A good example is provided by a recent study by Newsom and Palme of the abundances of molybdenum (Mo) and tungsten (W) in the Earth's mantle ${ }^{1}$, which lends strong support to multi-stage accretion models and severely limits the role played by metal and sulphide during the final stage of accretion.

As a result of the accretion processes, many elements are depleted in the Earth's mantle relative to their abundance in type 1 carbonaceous chondrites $(\mathrm{C} 1)$-believed to represent the most primitive SolarSystem material. The general sequence of depletion factors for lithophile elements in the Earth's mantle relative to chondrites (see figure) corresponds approximately to the predicted sequence of condensation of elements and phases from a cooling gas of solar composition. The regular distribution patterns suggest that the terrestrial depletion is related to the character of the source material within the Solar System and/or is a consequence of a selective condensation/volatilization process which occured during Earth's accretion. Depletion of the siderophile elements in the mantle reflects their preferential entry into the core (about one third of the Earth's mass) but the degree of loss of individual elements during accretion varies according to their volatility. The highly siderophile elements (platinum group elements, $\mathrm{Au}$ ) are depleted in the mantle by a factor of about 300 but maintain chondrite ratios relative to each other. A surprising feature of the figure is that the abundances of siderophile elements in the mantle are much greater than would be predicted by partition coefficients ${ }^{1}$ between iron and silicates expected to occur in the mantle. A multi-stage process of accretion and core formation is therefore required $^{3-5}$.

One viable model of that type has three main stages. In the first, there is accretion of 85 - 90 per cent of the Earth from reduced chondritic components with simultaneous formation of the iron core; all highly siderophile elements and most moderately siderophile elements are carried into the core, with minor retention of $\mathrm{Ni}, \mathrm{Co}$ and $\mathrm{FeO}$ in the silicate mantle. In the second stage, after initial core formation, 